\title{
ACTUATOR CELL MODEL OF THE 2D H-DARRIEUS WIND TURBINE
}

\author{
KRZYSZTOF RogOWSKI \\ Warsaw University of Technology, Institute of Aeronautics and Applied Mechanics, Warsaw, Poland \\ e-mail:krogowski@meil.pw.edu.pl
}

\begin{abstract}
This paper analyzes the instantaneous and averaged velocity field in the area of the Darrieus wind turbine. The analyzed two-dimensional rotor model consists of two NACA 0018 airfoils and a rotating shaft. The working parameters of the rotor correspond to moderate aerodynamic loads of the blades. The research has been carried out with an innovative method called the Actuator Cell Model. The initial results obtained were compared with the author's earlier results obtained with the SST k- $\omega$ model and with experimental studies taken from
\end{abstract} the literature.

Keywords: CFD, wind energy, vertical-axis wind turbine, Actuator Cell Model

\section{Introduction}

The flow past a Darrieus wind turbine rotor is very complex and difficult to analyze because the angle of attack varies with the azimuth. Existing simplified aerodynamic models based on the principle of conservation of momentum (Paraschivoiu, 2002) are currently insufficient (Ferreira et al., 2014). CFD modeling, taking into account the influence of the boundary layer, limits the application of these methods to 2D cases or individual simplified 3D models (Bangga et al., 2017). The modern market requires better tools for studying this type of wind turbines. However, such tools cannot be too expensive numerically.

In this work, an H-Darrieus wind turbine consisting of two straight blades and a rotating shaft is considered. A two-dimensional model for numerical research has been developed based on the paper (Tescione et al., 2014). The basic geometrical parameters of the rotor are given in Table 1.

Table 1. Geometrical characteristics of the rotor

\begin{tabular}{|l|c|}
\hline \multicolumn{1}{|c|}{ Parameter } & Value \\
\hline \hline Number of blades & 2 \\
\hline Rotor diameter $D=2 R[\mathrm{~m}]$ & 1 \\
\hline Airfoil & NACA 0018 \\
\hline Chord length $c[\mathrm{~m}]$ & 0.06 \\
\hline Shaft diameter $[\mathrm{m}]$ & 0.04 \\
\hline
\end{tabular}

According to Paraschivoiu (2002), a typical Darrieus wind turbine achieves its maximum aerodynamic efficiency (maximum rotor power coefficient) if the tangential velocity of the blade $V_{T}$ is 4-5 times higher than the wind speed $V_{0}$. The tangential velocity of the rotor blade depends on the rotor radius $R$ and its angular velocity $\omega, V_{T}=\omega R$. The $V_{T} / V_{0}$ ratio is called the tip speed ratio (TSR). At tip speed ratios of 4-5, the local angles of attack $\alpha$ (the angle between the chord line and the relative velocity $W$ ) often do not exceed the so-called critical angles of 
attack (Fig. 1). For lower tip speed ratios, the rotor blades experience deep dynamic stall. This means that rotors operating in such a regime can be studied using the classic CFD approach with satisfactory accuracy. Therefore, in this work, the rotor operating at the tip speed-ratio of 4.5 has been chosen for analysis. Numerical studies were carried out for flow parameters corresponding to wind tunnel tests (Tescione et al., 2016). The flow velocity $V_{0}$ was $9.3 \mathrm{~m} / \mathrm{s}$, whereas the turbulence intensity level amounted to $0.24 \%$.

(a)

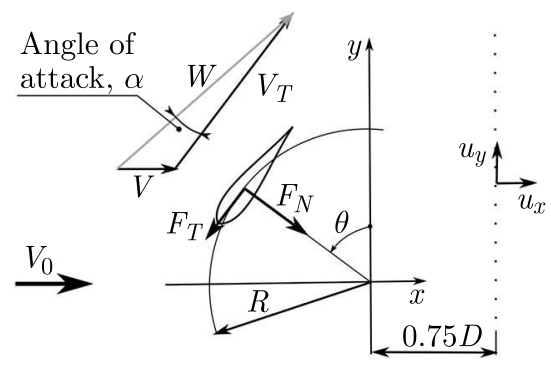

(b)

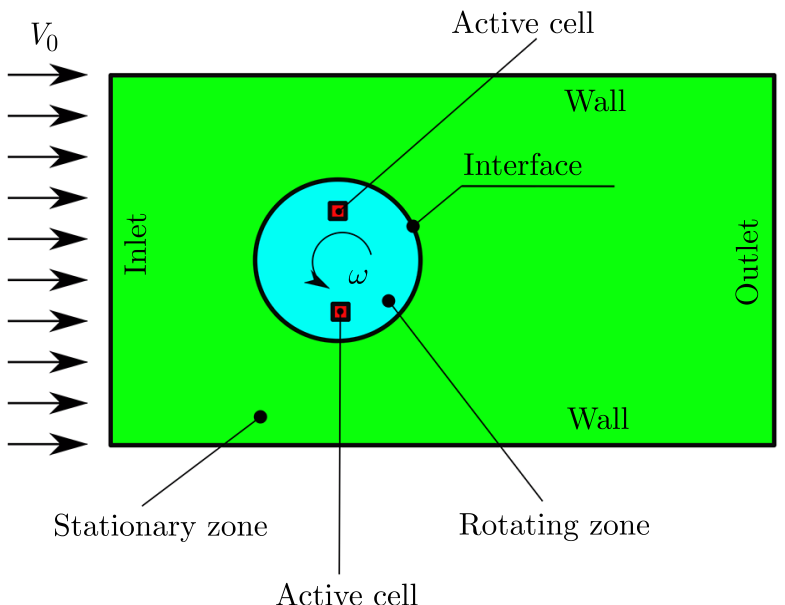

Fig. 1. Vectors of aerodynamic forces and velocities (a) and ACM concept (b)

The idea of the Actuator Cell Model (ACM) lies in the possibility of studying the velocity field in the rotor area at a given function of aerodynamic blade loads. The previous two publications of the author showed the effectiveness of this method for the case of a one-bladed H-Darrieus rotor (Rogowski, 2018; Rogowski and Rogowska, 2018). In this article, the latest particle image velocimetry (PIV) studies by Tescione et al. (2016) are used to compare the results obtained with the ACM model.

\section{Numerical modeling}

The Actuator Cell Model utilizes a finite volume grid; however, the presence of rotor blades is not modeled in the traditional way. The computational domain does not take into account the shape of the blades, and the discrete model (mesh) does not take into account the structural grid in the vicinity of the airfoil edge in order to determine the boundary layer.

In the ACM approach presented in this paper, the aerodynamic blade loads, their normal and tangential components, are known functions of the rotor rotation angle. The ACM method also allows the determination of these functions using the aerodynamic characteristics, lift and drag coefficients of the airfoils. The purpose of this test, however, is to determine the velocity field in the vicinity of the rotor based on known tangential and normal force functions calculated using the classic CFD approach from Rogowski (2019).

The ACM approach utilizes the known ANSYS Fluent technique - sliding mesh. Thanks to this, it is possible to study the unsteady flow around the rotor. In order to introduce the aerodynamic forces of the blades, additional mesh zones are created consisting of one grid cell. For each moment of time corresponding to the angular position of the rotor (azimuthal position $\theta$, Fig. 1), the aerodynamic loads are introduced into these additional mesh zones, so-called the active cells. Components of the aerodynamic force projected on the axes of the Cartesian coordinate system respected by ANSYS Fluent are introduced into Navier-Stokes equations as so-called momentum sources, which are defined as 


$$
S_{x, y}=\frac{F_{x, y}}{V_{c}}
$$

where $V_{c}$ is the mesh cell volume whereas $F_{x}$ and $F_{y}$ are the aerodynamic blade loads. The ACM approach is described in more detail in two publications: Rogowski (2018) and Rogowski and Rogowska (2018). The aerodynamic blade load functions are taken from Rogowski (2019).

\section{Results and discussion}

Figure 1 presents instantaneous fields of velocity components obtained using the k- $\omega$ SST turbulence model (Rogowski, 2019) and the ACM approach. The obtained results were also compared with the experimental results obtained with the PIV technique (Tescione et al., 2016). The CFD results present good agreement between each other. Differences between the numerical and experimental results arise from the simplifications of the 2D model used.

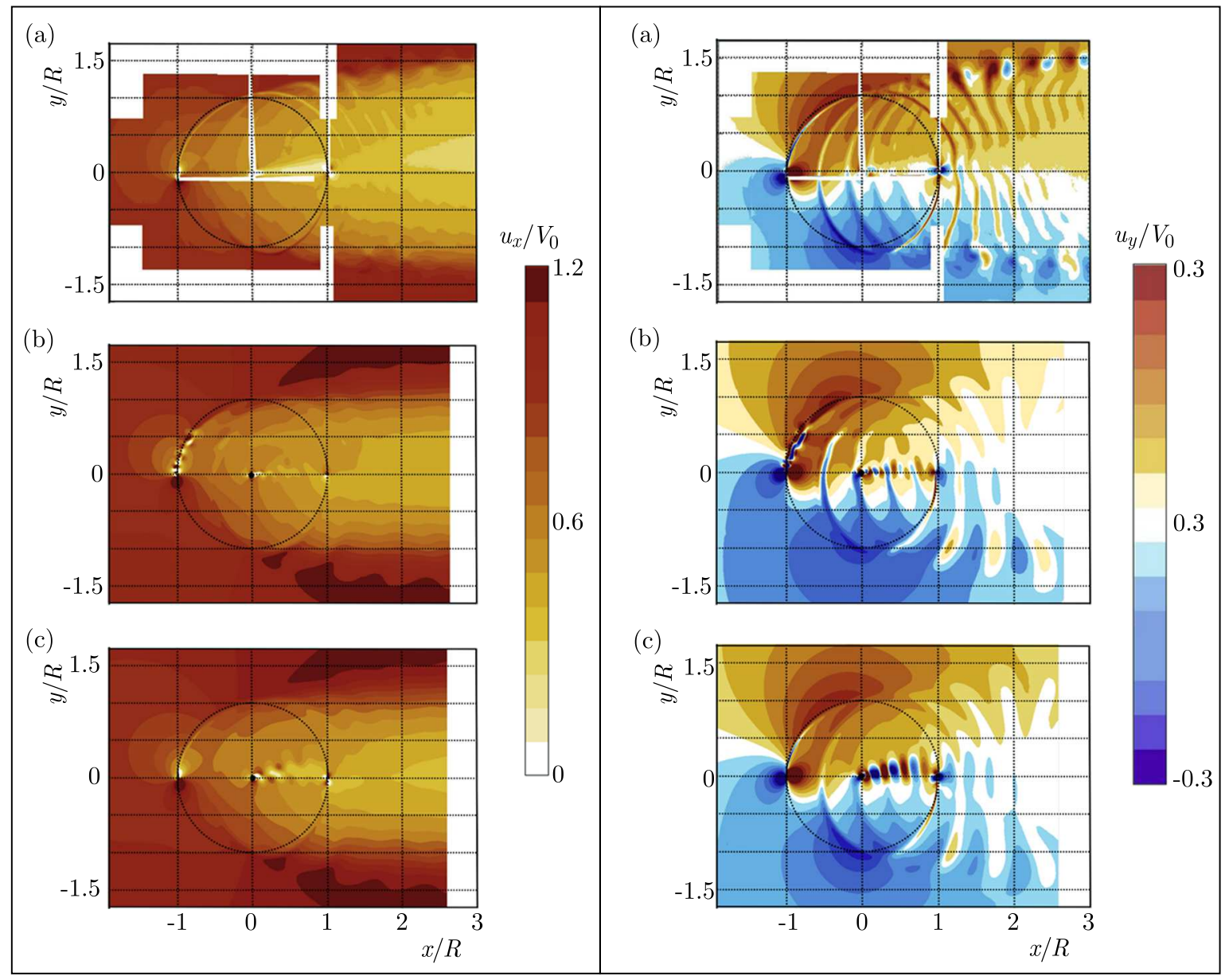

Fig. 2. Instantaneous velocities: the component parallel to the wind direction $u_{x}$ (on the left) and the component perpendicular to the wind direction $u_{y}$ (on the right). The results are given for: (a) PIV (Tescione et al., 2014), (b) ACM, and (c) full CFD with the k- $\omega$ SST turbulence model (Rogowski, 2019)

Figure 3 presents velocity profiles averaged over one rotor revolution given at the distance of $0.75 \mathrm{D}$ downstream behind the rotor. The obtained numerical results are also compared with the independent velocity profiles by Lam and Peng (2016) who used the k- $\omega$ SST turbulence approach. The obtained agreement in the results is satisfactory. 

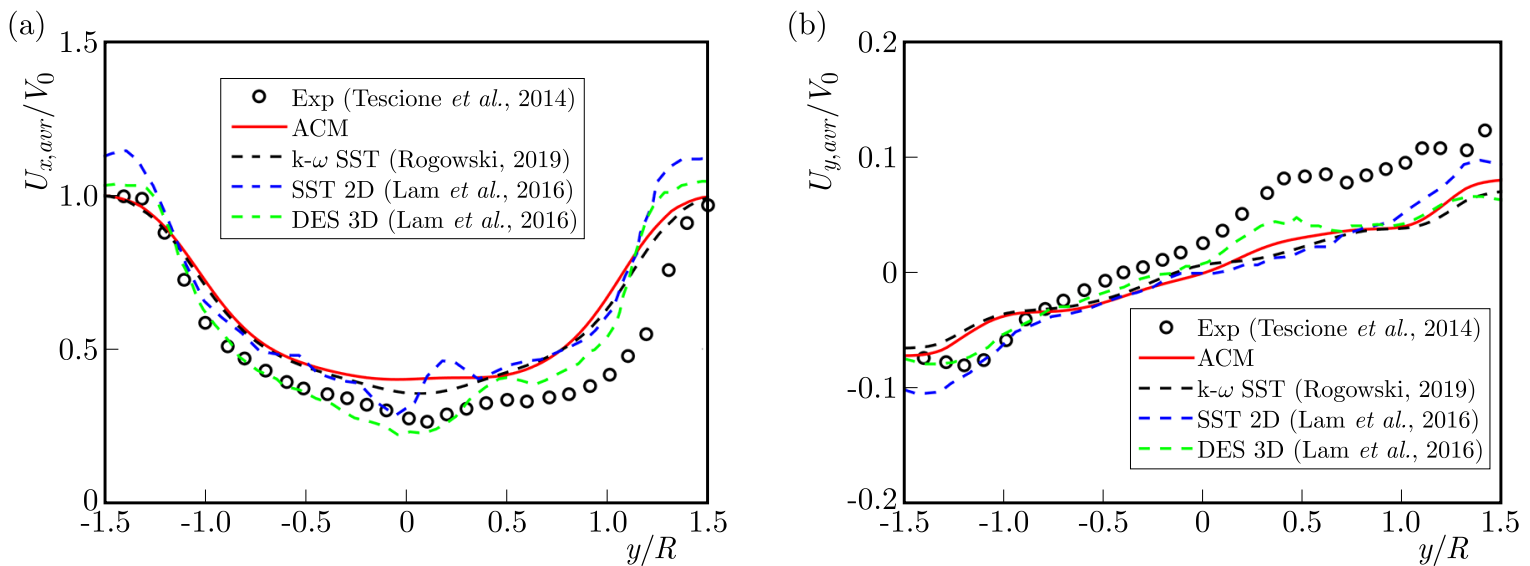

Fig. 3. The components of the flow velocity at $0.75 D$ behind the rotor averaged for one full rotation of the rotor: the component parallel to the wind direction $U_{x, \text { avr }}$ (a) and the component perpendicular to the wind direction $U_{y, a v r}(\mathrm{~b})$. Velocity profiles are normalized by the wind speed $V_{0}$

\section{Conclusions}

The ACM technique allows one to correctly calculate the velocity field in the rotor area. Discrepancies in the results are similar to those obtained using the classic CFD approach. In the numerical model, additional structural elements of the rotor, such as struts that attach the blades to the rotating shaft, have not been taken into account. These elements can have a significant impact on the flow field.

\section{References}

1. Bangga G., Hutomo G., Wiranegara R., Sasongko H., 2017, Numerical study on a single bladed vertical axis wind turbine under dynamic stall, Journal of Mechanical Science and Technology, 31, 1, 261-267

2. Ferreira C.S., Madsen H.A., Barone M., Roscher B., Deglaire P., Arduin I., 2014, Comparison of aerodynamic models for vertical axis wind turbines, Journal of Physics: Conference Series, 524, 1, 012125

3. Lam H.F., Peng H.Y., 2016, Study of wake characteristics of a vertical axis wind turbine by twoand three-dimensional computational fluid dynamics simulations, Renewable Energy, 90, 386-398

4. Paraschivoiu I., 2002, Wind Turbine Design: with Emphasis on Darrieus Concept, Polytechnic International Press, Canada

5. Rogowski K., 2018, The actuator cell model for the Darrieus wind turbine, Journal of Physics: Conference Series, 1101, 012028

6. Rogowski K., 2019, CFD Computation of the H-Darrieus wind turbine - the impact of the rotating shaft on the rotor performance, Energies, 12, 13

7. Rogowski K., Rogowska K., 2018, Velocity field around Darrieus wind turbine rotor using actuator cell model and other CFD methods, Task Quarterly, 22, 3

8. Tescione G., Ferreira C.S., van Bussel G.J.W., 2016, Analysis of a free vortex wake model for the study of the rotor and near wake flow of a vertical axis wind turbine, Renewable Energy, $\mathbf{8 7}, 552-563$

9. Tescione G., Ragni D., He C., Ferreira C.S., van Bussel G.J.W., 2014, Near wake flow analysis of a vertical axis wind turbine by stereoscopic particle image velocimetry, Renewable Energy, 70, 47-61, DOI: 10.1016/j.renene.2014.02.042 